\title{
SOLID-PHASE EXTRACTION OF NEONICOTINOIDS RESIDUE FROM WATER: COMPARISON BETWEEN EXTRACTION CARTRIDGES
}

\author{
Gorica Vuković ${ }^{1}$, Dušan Marinković ${ }^{2}$, Aleksandra Petrović ${ }^{2}$, Bojan Konstantinović ${ }^{2}$, \\ Tijana Stojanović ${ }^{2}$, Nikola Puvača ${ }^{3}$, Vojislava Bursić ${ }^{2 *}$ \\ ${ }^{1}$ Institute of Public Health of Belgrade, Bulevar despota Stefana 54A, Serbia \\ ${ }^{2}$ University of Novi Sad, Faculty of Agriculture, Dositeja Obradovića 2, Novi Sad, Serbia \\ ${ }^{3}$ University Business Academy, Faculty of Economics and Engineering Management, Novi Sad, Serbia
}

*E-mail of corresponding author: bursicv@polj.uns.ac.rs

\begin{abstract}
The Commission Implementing Decision (EU) 2018/840 established the watch list of substances for Union-wide monitoring in the field of water policy pursuant to Directive 2008/105/EC and Commission Implementing Decision (EU) 2015/495. This Decision establishes additional substances and their maximum acceptable method detection limits including neonicotinoids (9-500 $\mathrm{ng} / \mathrm{L}$ ). During the pesticides extraction from water, the solid-phase extraction (SPE) column was used. Bakerbond spe ${ }^{\mathrm{TM}}$ SDB-1 SPE and Bond Elut Plexa were evaluated for the SPE of neonicotinoids (imidacloprid, thiamethoxam, acetamiprid, thiacloprid and clothianidin) from water. The comparation of the results of these two types of columns, was performed by spiking water samples at two levels $(0.05$ and $0.1 \mu \mathrm{g} / \mathrm{L})$ in three replicates. The Bakerbond ${ }^{\mathrm{TM}}$ column quantitatively adsorbed these pesticides, with the obtained recoveries: $36.9 \%$ for imidacloprid, $43.2 \%$ for thiacloprid, 119.3 $\%$ for clothianidin, $64.6 \%$ for acetamiprid and $53.6 \%$ for thiamethoxam. Using Bond Elut Plexa the obtained recoveries were $66.9 \%$ for imidacloprid, $72.9 \%$ for thiacloprid, $103.4 \%$ for clothianidin, $67.4 \%$ for acetamiprid and $45.2 \%$ for thiamethoxam. Both colums have low recovery values for thiametoxam, while the highest values were obtained by Bond Elut Plexa for thiacloprid, imidacloprid and acetamiprid. Great recoveries were achieved for clothianidin using both SPE columns. Keywords: neonicotinoids, SPE extraction, water, LC-MS/MS.
\end{abstract}

\begin{tabular}{c} 
Received: 15.07.2019. / Accepted: 27.11.2019. \\
Published online: 09.12.2019. \\
\hline
\end{tabular}

Original scientific paper

\section{INTRODUCTION}

Water is one of the essential resources for life and its multiple uses are indispensable for a series of activities, such as agriculture, generation of energy, public and industrial supply, among others (Stojanović et al. 2017). Water covers about $70 \%$ of the earth, but only $3 \%$ of the world's water is fresh and twothirds of that amount is tucked away in frozen glaciers or otherwise unavailable for our use (Lomsadze et al. 2018). The problem of watercourses pollution has become the main topic of discussion among numerous scientists and experts in the field of environmental protection. Unfortunately, this problem was observed relatively late (at the end of the 1980s), after the chemicals in water caused the disappearance of many aquatic organisms. Particular attention is paid to pesticides that can migrate to surface and groundwater after the application to plants or soil (Ismail et al. 2012). Very often, agricultural producers, after finishing spraying, throw away empty containers into the local channels or leave them near the field, which is another source of pollution (Bursić et al. 2013). Although pesticides are considered to be extremely toxic substances in the environment, there is very little information about their distribution and use in Serbia (Antić et al. 2013).

Neonicotinoids are nowadays the most widely used insecticides in the world and include imidacloprid, thiamethoxam, acetamiprid, thiacloprid and clothianidin, as well as a metabolite 6-chloro nicotinic acid (Bursić et al. 2016). Despite these potential advantages, nowadays, the use of neonicotinoids is a matter of concern due to their high mobility in plants and environmental matrices, having been detected in the surface water samples, obtained in the vicinity of agricultural areas, from different regions of the planet (Casadol et al. 2016). Apart from their direct impact in honey production, their toxicity for bees presents a threat for the biodiversity of ecosystems. Commission Implementing Decision (EU) (2018) 2018/840 of 5 June 2018 established a watch list of substances for Union-wide monitoring in the field of water policy pursuant to Directive 2008/105/EC of the European Parliament and of the Council and repealing Commission Implementing Decision (EU) 2015/495. This Decision establishes additional substances and their maximum acceptable method detection limits like oxadiazon (8.8 ng/L), methiocarb (10 ng/L) and neonicotinoids $(9-500 \mathrm{ng} / \mathrm{L})$.

Among the different pre-treatment approaches, SPE (solid-phase extraction) offers a good compromise between robustness, rapidity, convenience, clean-up efficiency, scope for automation and solvent consumption 
and is, therefore, ideally suited to routine analysis (Xie et al. 2011). SPE columns, mostly OASIS HLB cartridges, are widely used for neonicotinoids residue analysis for different samples such as vegetable and fruits (Obana et al. 2003; Xie et al. 2011), cottonseed cake (Mohan et al. 2010) and wine (Rodriguez-Cabo et al. 2016).

The low maximum residue limits (MRLs) have fostered the development of more powerful sensitive analytical methods to meet the requirements in the complex samples, such as food, water and soil. In this sense, liquid chromatography-tandem mass spectrometry (LC-MS/MS) with triple quadrupole in multiple reaction monitoring (MRM) mode has become so far, the most widely used technique for the quantitation of neonicotinoid insecticides in different matrices as reported extensively in the literature.

During the imidacloprid, thiamethoxam, acetamiprid, thiacloprid and clothianidin (Figure 1) extractions from water, the SPE column was used. This study reports the comparison between extraction cartridges: Bakerbond spe ${ }^{\mathrm{TM}}$ SDB-1 Solid Phase Extraction Columns (J.T. Baker ${ }^{\mathrm{TM}}$, USA) and Bond Elut Plexa (Agilent Technologies, USA) in the terms of neonicotinoid insecticide recoveries from water using LC-MS/MS.<smiles>CC(=NC#N)N(C)Cc1ccc(Cl)nc1</smiles>

Acetamiprid<smiles>O=[N+]([O-])NC1=NCCN1Cc1ccc(Cl)nc1</smiles>

Imidacloprid<smiles>CN1COCN(CC2CN=C(Cl)S2)C1=[N+]([O-])[O-]</smiles>

Thiamethoxam<smiles>N#CN=C1SCCN1Cc1ccc(Cl)nc1</smiles>

Thiacloprid<smiles>C/N=C(/NCc1cnc(Cl)s1)N[N+](=O)[O-]</smiles>

Clothianidin

Figure 1. Chemical structures of five neonicotinoid pesticides (Ghanim \& Ishaaya 2011)

\section{MATERIALS AND METHODS}

\subsection{Standards, solvents and sorbents}

HPLC grade acetonitrile and methanol were obtained from Merck (Darmstadt, Germany). Formic acid (purity $98 \%, w / w)$ was from Sigma-Aldrich (Steinheim, Germany). The water used was purified with a Milli-Q water purification system from Millipore (Bedford, MA, USA). Cartridges used for solid-phase extraction were Bakerbond spe ${ }^{\mathrm{TM}}$ SDB-1 Solid Phase Extraction Columns (200 mg, 6 mL - J.T. BakerTM, USA) and Bond Elut Plexa (60 mg, $3 \mathrm{~mL}$ - Agilent Technologies, USA). PTFE syringe filter (Millex LCR, Millipore, Milford, MA, USA).

Individual standard pesticide stock solutions $(\approx 0.50 \mathrm{mg} / \mathrm{mL})$ were prepared in methanol and stored at $-20{ }^{\circ} \mathrm{C}$. Working standard (multi component solution $-10 \mu \mathrm{g} / \mathrm{mL}$ ) was prepared by diluting stock solution with the chromatographic mobile phase (acetonitrile/water $(0.1 \%$ formic acid) 30:70, v/v) and was used for spiking tap water samples.

\subsection{Spiking samples}

The recoveries were done at two concentration levels $(0.05$ and $0.1 \mu \mathrm{g} / \mathrm{L})$ in three replicates. The method precision is expressed as the repeatability (RSD \%) based on the recovery experiments (SANTE/11813/2017).

\subsection{Solid-phase extraction}

Before the use, the activation Bakerbond spe ${ }^{\mathrm{TM}} \mathrm{SDB}-1$ and Bond Elut Plexa were conditioned with $5 \mathrm{~mL}$ of methanol and $5 \mathrm{~mL}$ of deionized water. The samples were percolated through the cartridges and left to flow through 
under the action of gravity. The cartridges were eluted with $5 \mathrm{~mL}$ of methanol and the eluate was evaporated to dryness on a steam of nitrogen. The dried extract was reconstituted in $0.2 \mathrm{~mL}$ of mobile phase, vortex mixed for $60 \mathrm{~s}$. The final solution was filtered through a $0.22 \mu \mathrm{m}$ PTFE syringe filter and injected in LC-MS/MS.

\subsection{LC-MS/MS conditions}

An Agilent series 1200 HPLC system (Agilent Technologies) equipped with a G1312B binary pump, a G1367D auto sampler, a G1379B degasser, a G1316B column compartment thermostat, The HPLC system was coupled to an Agilent triple quadrupole mass spectrometer (6410B) coupled to an electrospray ionization source (ESI+). A Zorbax XDB C18 column (50x4.6 mm, $1.8 \mu \mathrm{m}$ particle size) from Agilent (San Jose, CA, USA) was employed for the separation. The chromatographic determination of neonicotinoids was carried out employing a binary mobile phase with methanol $(0.1 \% \mathrm{HCOOH}, \mathrm{v} / \mathrm{v}-\mathrm{A})$ and an aqueous solution of formic acid $(0.1 \%$, v/v - B). A gradient elution started at $60 \%$ of B at the flow rate of $0.4 \mathrm{~mL} / \mathrm{min}$. This composition was reduced to 30 $\% \mathrm{~B}$ in $10 \mathrm{~min}$, and held for $5 \mathrm{~min}$. The system was equilibrated during $5 \mathrm{~min}$. The injection volume was $5 \mu \mathrm{L}$ and column temperature was kept at $30^{\circ} \mathrm{C}$. The ESI source values were as follows: drying gas (nitrogen) temperature $350{ }^{\circ} \mathrm{C}$, drying gas flow rate $10 \mathrm{~L} / \mathrm{min}$, nebulizer pressure $40 \mathrm{psi}$ and capillary voltage $4000 \mathrm{~V}$. The detection was performed using the multiple reactions monitoring mode (MRM). The Agilent MassHunter software (version B.06.00 Agilent Tehnologies, 2012) was used for the optimization and quantification.

\section{RESULTS AND DISCUSSION}

The LC-MS/MS (ESI+) fragmentation of the protonated molecular ions of the investigated neonicotinoid insecticides, which yielded two product ions, respectively given in Table $\mathbf{1 .}$

Table 1. MRM transitions, fragmentation (FRAG), colision energies (CE) and retention times $\left(\mathrm{t}_{\mathrm{R}}\right)$

\begin{tabular}{|c|c|c|c|c|c|}
\hline PESTICIDE & MW & TRANSITION $(\mathbf{m} / \mathbf{z})$ & CE $(\mathbf{V})$ & FRAG $(\mathbf{V})$ & t$_{\mathbf{R}}(\mathbf{m i n})$ \\
\hline Acetamiprid & 222.67 & $\begin{array}{c}223.1 \rightarrow 55.7 \\
223.1 \rightarrow 125.8\end{array}$ & $\begin{array}{c}15 \\
20\end{array}$ & 100 & 16.288 \\
\hline \multirow{2}{*}{ Imidacloprid } & 255.66 & $\begin{array}{l}256.0 \rightarrow 174.6 \\
256.0 \rightarrow 208.7\end{array}$ & $\begin{array}{c}20 \\
15\end{array}$ & 100 & 9.176 \\
\hline Clothianidin & 249.68 & $\begin{array}{l}250.0 \rightarrow 132.1 \\
250.0 \rightarrow 169.1\end{array}$ & $\begin{array}{l}15 \\
10\end{array}$ & 90 & 12.696 \\
\hline Thiamethoxam & 291.71 & $\begin{array}{l}292.0 \rightarrow 181.0 \\
292.0 \rightarrow 211.0\end{array}$ & $\begin{array}{l}20 \\
10\end{array}$ & 80 & 7.179 \\
\hline Thiacloprid & 252.72 & $\begin{array}{l}253.0 \rightarrow 126.0 \\
253.0 \rightarrow 186.0\end{array}$ & $\begin{array}{c}20 \\
10\end{array}$ & 110 & 19.240 \\
\hline
\end{tabular}

"MW-molecular weight

The average recoveries were calculated from the gained recovery values of spiking water samples at concentrations of 0.05 and $0.1 \mu \mathrm{g} / \mathrm{L}$, in three replicates. Compared average recoveries using Plexa and JBT columns are given in Table 2, while the graphic recovery comparison of investigated neonicotinoids is shown in Figure 2.

Table 2. Obtained average recovery

\begin{tabular}{|c|c|c|c|c|c|c|c|c|c|c|}
\hline & \multicolumn{2}{|c|}{ THIAMETHOXAM } & \multicolumn{2}{l|}{ CLOTHIANIDIN } & \multicolumn{2}{c|}{ THIACLOPRID } & \multicolumn{2}{c|}{ IMIDACLOPRID } & \multicolumn{2}{c|}{ ACETAMIPRID } \\
\cline { 2 - 13 } & Plexa & JTB & Plexa & JTB & Plexa & JTB & Plexa & JTB & Plexa & JTB \\
\hline $\begin{array}{c}\text { Average } \\
\text { recovery, \% }\end{array}$ & 45.2 & 53.6 & 103.4 & 119.3 & 72.9 & 43.2 & 66.9 & 36.9 & 64.3 & 66.9 \\
\hline SDEV & 1.28 & 8.09 & 18.74 & 14.08 & 7.91 & 4.23 & 13.82 & 20.34 & 3.94 & 1.67 \\
\hline RSD, \% & 2.84 & 15.07 & 18.12 & 11.81 & 10.84 & 9.79 & 20.64 & 55.11 & 6.12 & 2.49 \\
\hline
\end{tabular}




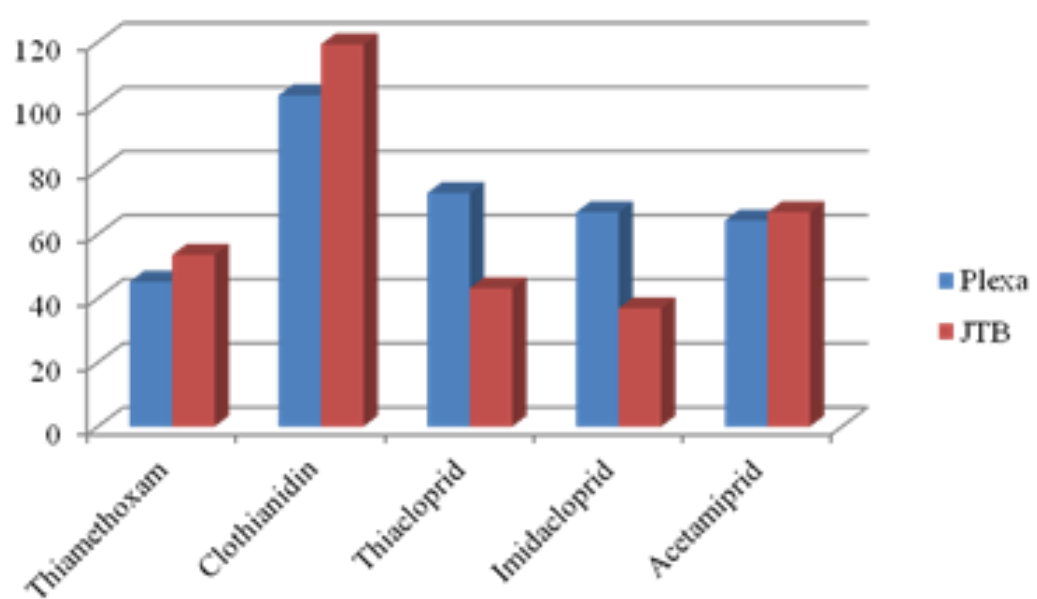

Figure 2. Graphic comparison of obtained average recovery

The LC-MS/MS total ion chromatogram (TIC) from a blank water sample spiked with all the analytes at the contrentation level of $0.1 \mu \mathrm{g} / \mathrm{L}$ is shown in Figure 3. The retention times of the investigated neonicotinoide pesticides are given in Table 1. First peak at $7.179 \mathrm{~min}$ was the peak of thiamethoxam, followed by imidacloprid, clothianidin, acetamiprid and thiacloprid.
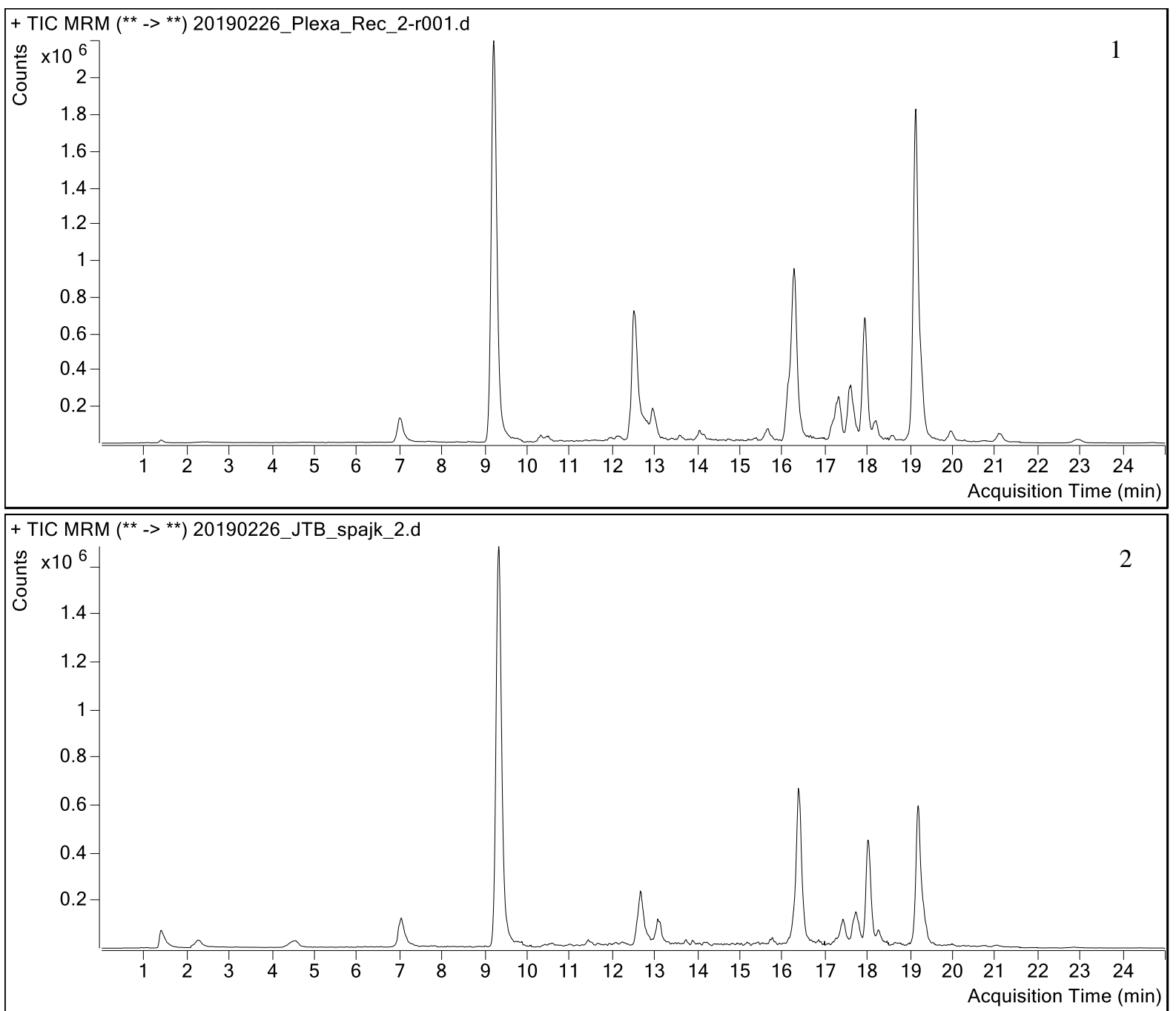

Figure 3. TIC chromatogram of spiking sample obtained by Plexa (1) and JBT column (2) 
In order to statistically compare the two types of columns, the null hypothesis is defined, which implies that there are no significant differences in the means of each sample. The hypothesis was tested using t-test: TwoSample Assuming Equal Variances and F-test Two-Sample for Variances, and the results are presented in Table 3. The significant differences in the precision of the results for two data sets were detected only for imidacloprid, so the null hypothesis was accepted in this case. The performances of the tested column types demonstrated significant differences in the results accuracy for thiacloprid (T-test) and the precision of the results for imidacloprid (F-test).

Table 3. The results of T- and F-test

\begin{tabular}{|c|c|c|c|c|c|}
\hline & THIAMETHOXAM & CLOTHIANIDIN & THIACLOPRID & IMIDACLOPRID & ACETAMIPRID \\
\hline $\mathrm{F}$ & 0.025 & 1.77 & 3.50 & 0.46 & 5.58 \\
\hline F Critical & 0.157 & 6.39 & 9.12 & 0.11 & 9.28 \\
\hline F<Fcrit. & Yes $^{1}$ & Yes & Yes & No $^{3}$ & Yes \\
\hline t Stat & -2.30 & -1.52 & 6.74 & 2.44 & 0.09 \\
\hline $\mathrm{t}_{\text {Critical two-tail }}$ & 2.31 & 2.31 & 2.36 & 2.57 & 2.36 \\
\hline $\mathrm{t}_{\text {stat. }<\text { tcrit. }}$ & Yes & Yes & $\mathrm{No}^{2}$ & Yes & Yes \\
\hline
\end{tabular}

${ }^{1}$ Since the $\mathrm{p}$ - value is less than our alpha (0.05). the null hypothesis that there are no significant differences in each sample means is rejected

${ }^{2}$ Significant difference in the accuracy results for two data sets

${ }^{3}$ Significant difference in the precision of the results for two data sets

\section{CONCLUSION}

The comparison of the results of Bakerbond ${ }^{\mathrm{TM}}$ column with styrene-divinylbenzene (SDB) copolymer/ $6 \mathrm{~mL}$ capacity, $200 \mathrm{mg}$ bed weight and Bond Elut Plexa $(60 \mathrm{mg}, 3 \mathrm{~mL})$ with unique polymeric architecture, a no retentive, hydroxylated, amide-free surface, and a nonpolar PS-DVB core for retaining small molecules column types was performed by spiking water samples at two levels $(0.05$ and $0.1 \mu \mathrm{g} / \mathrm{L})$ in three replicates. The obtained statistical data using t- and F-test, indicate the presence of the significant differences in the results for two data sets in the results accuracy for thiacloprid and the precision of the results for imidacloprid (T-test and F-test, respectively).

\section{ACKNOWLEDGMENTS}

The authors acknowledge the financial support of the Ministry of Education and Science. Republic of Serbia. Project Ref. III43005.

\section{REFERENCES}

Antić N, Radišić M, Radović T, Vasiljević T, Grujić S, Petković A, Dimkić M, Laušević M (2013) Pesticide residues in the Danube River Basin in Serbia-a survey in 2009-2011. CLEAN - Soil. Air. Water. 43, 2, $197-204$.

Bursić V, Gvozdenac S, Vuković G, Pucarević M, Lazić S, Vuković S, Zeremski T, Inđić D (2013) Comparative study of pesticide residue levels in water from irrigation canal with LC-MS/MS and biological methods. $3^{\text {rd }}$ International Conference of Ecology "Essays on Ecosystem and Environmental Research". May 31June 5.2013. Tirana. Albania. Proceeding book, 870-874.

Bursić V, Vuković G, Stojanović Z, Gvozdenac S, Zeremski T, Meseldžija M, Petrović A (2016) Implementacija direktive EU 495/2015 u praćenju kvaliteta vode Dunava. XXI Savetovanje o biotehnologiji sa međunarodnim učešćem. Čačak. Srbija. Zbornik radova. 21. 23, 359-365.

COMMISSION IMPLEMENTING DECISION (EU) 2018/840 of 5 June 2018 establishing a watch list of substances for Union-wide monitoring in the field of water policy pursuant to Directive 2008/105/EC of the European Parliament and of the Council and repealing Commission Implementing Decision (EU) 2015/495.

Ghanim M, Ishaaya I (2011) Insecticides with Novel Modes of Action Mechanism and Resistance Management. In: Tolerance to Environmental Contaminants. Amiard-Triquet. C.. Rainbow. P. S. and Romeo. M. (eds.). CRC Press. chapter: 16. 
Ismail BS, Humaira Haron S, Talib Latif M (2012) Pesticide pesidue levels in the surface water of the irrigation canals in the muda irrigation Scheme Kedah. Malaysia. International Journal of Basic \& Applied Sciences IJBAS. 12. 6. 85-90.

Lomsadze Z. Makharadze K. Gamezardashvili D, Pirtskhalava R (2018) Problems of drinking water in Kakheti region. Journal of Agronomy, Technology and Engineering Management JATEM, 1, 1, 57-64.

Mohan C, Kumar Y, Madan J, Saxena N (2010) Multiresidue analysis of neonicotinoids by solid-phase extraction technique using high-performance liquid chromatography. Environ. Monit. Assess. 165. 573-576. DOI 10.1007/s10661-009-0968-8.

Obana H, Okihashi M, Akutsu K, Kitagawa Y, Hori S (2003) Determination of neonicotinoid pesticide residues in vegetables and fruits with solid phase extraction and liquid chromatography mass spectrometry. J. Agric. Food Chem. 51. 2501-2505.

Rodriguez-Cabo T. Casado J. Rodriguez I. Ramil M. Cela R (2016) Selective extraction and determination of neonicotinoid insecticides in wine by liquid chromatography-tandem mass spectrometry. Journal of Chrom. A. 1460, 9-15. DOI 10.1016/j.chroma.2016.07.004

SANTE/11813/2017: Method validation and quality control procedures for pesticide residues analysis in food and feed.

Stojanović Z, Deršek Timotić I, Bursić V, Špirović-Trifunović B, Vuković G, Zeremski T (2017) Screening of neonicotinoids in surface water samples by liquid chromatography quadropole time-of-flight mass spectrometry. Proceedings of the 23rd International Symposium on Analytical and Environmental Problems, Szeged, Hungary, 329-333.

Xie W, Han C, Qian Y, Ding H, Chen X, Xi J (2011) Determination of neonicotinoid pesticides residues in agricultural samples by solid-phase extraction combined with liquid chromatography-tandem mass spectrometry. J. Chrom. A. 1218, 4426-4433. 